ISSN 1112-9867

\title{
TEST-RETEST RELIABILITY OF KNEE KINEMATICS MEASUREMENT DURING GAIT WITH 3D MOTION ANALYSIS SYSTEM
}

\author{
F. Z. Fairus ${ }^{1,2}$, N. A. M. Nordin ${ }^{2, *}$ and D. K. A $\operatorname{Singh}^{2}$ \\ ${ }^{1}$ Faculty of Sports Sciences and Coaching, Universiti Pendidikan Sultan Idris, Perak, \\ Malaysia \\ ${ }^{2}$ Faculty of Health Sciences, Universiti Kebangsaan Malaysia, Kuala Lumpur, Malaysia.
}

Published online: 10 November 2017

\begin{abstract}
Restoration of gait symmetry following anterior cruciate ligament reconstruction (ACLR) is crucial to minimize the risk of joint degeneration. To achieve this, it is essential that the chosen measurement method can accurately assess knee kinematics and detect the changes in multi-planes of motion. However to date, limited study is available on repeatability of the multi-planes knee angle measurements particularly among male patients post ACLR. The purpose of this study was to assess the test-retest reliability of knee kinematic measurements using three-dimensional (3D) motion analysis system during gait in post ACLR patients. Eight patients with mean (SD) age 28.89 (4.0) years, 5.82(4.07) months post ACLR were recruited from a tertiary hospital of Kuala Lumpur. All patients undergone two sessions of knee joint angles measurement during gait at four hours interval, for the injured and the non-injured knees. Angles in the sagittal, frontal and transverse planes during initial contact phase of gait that derived from the two measurement sessions were compared.
\end{abstract}

Author Correspondence, e-mail: norazlin8@ukm.edu.my

doi: http://dx.doi.org/10.4314/jfas.v9i6s.92 
The results showed high test-retest reliability of the measurement in sagittal and transverse planes; the intraclass correlation coefficient (ICC) was $0.97(95 \% \mathrm{CI}: 0.84-0.99)$ and 0.96 (95\% CI: 0.83-0.99) respectively, and moderate test-retest reliability for the measurements in frontal planes, with ICC 0.44 (95\% CI: -0.32-0.86). The study findings suggest that multi-planes knee angle measurements during initial contact phase of gait could reliably be measured using a 3D motion analysis system. Further research may focus on knee kinematics measurements at other phases of gait.

Keywords: biomechanics, reliability, walking, knee, anterior cruciate ligament reconstruction

\section{INTRODUCTION}

ACL injury is a musculoskeletal problem with debilitating effect to the injured individual. It requires not only treatment at a higher cost and longer duration of rehabilitation, but may cause increased risk of knee osteoarthritis in a later years [1-3]. Anterior cruciate ligament reconstruction (ACLR) is one of the most common surgeries following ACL injury. An Australian study reported that over 50,187 ACL reconstructions were performed over a five-year period between 2003 and 2008 [1]. The main goal of ACLR is to restore knee stability and expedite the return to sports [2,4]. An early promotion of knee stability could reduce the risk of meniscus and cartilage tear, and prevent the occurrence of knee osteoarthritis [2,3]. Following ACLR, patients are required to participate in a comprehensive but specific rehabilitation program for duration of 4 months up to 1 year. Specific rehabilitation protocols are implemented at a high intensity and substantial frequency and duration. However, despite intensive rehabilitation, ACLR patients demonstrate alterations of knee joint angle, particularly during gait $[5,6]$. These alterations have been linked to the development of knee osteoarthritis among ACLR patients [5,7]. A gait analysis, looking specifically at knee kinematics is therefore important to detect the alterations of the knee angles during the early stage of rehabilitation.

Movement of knee joints during gait occurs in several planes of motion, namely sagittal, frontal and transverse planes [8]. It is noted in a previous study that alterations of knee angle during gait occurs in multiple planes of movement [9]. Although reliability of measurements of knee kinematic during gait has been reported in previous studies [10-12], little information 
is available on multi-planes knee angle measurements among ACLR patient. Labbe and colleagues [12] in a study of knee joint angle measurement during gait analysis among healthy individuals, reported high repeatability of measurements in all planes of motion. On a contrary, other similar studies reported otherwise, particularly on the movement out of sagittal plane $[10,11]$. In these studies, 3D motion analysis system was used. Characteristic of the participants, laboratory settings, procedure adopted, disease severity, and assessor experiences may also affect the reliability of knee angle measurement [13]. In addition, kinematics of knee during various functional tasks including gait are also known to be different by gender $[14,15]$. Therefore, further studies that assess gender specific reliability of knee measurements are warranted to establish the measurement method and effectively interpret post-ACLR patients' gait performance. The aim of this study was to determine the reliability of knee angle measurement using 3D motion analysis system in sagittal, frontal and transverse planes of motion during among ACLR male patients.

\section{METHODOLOGY}

\subsection{Study Design and Setting}

This was a cross-sectional laboratory study conducted at the Faculty of Health Sciences, Universiti Kebangsaan Malaysia (UKM). The UKM Research Ethics Committee approved the study protocol (Code Project: NN-072-2014).

\subsection{Participants}

Eight post-ACLR male patients were recruited from the orthopaedic clinic of the Universiti Kebangsaan Malaysia Medical Centre (UKMMC) based on the following inclusion criteria: (a) age between 19 and 45 years; (b) ACLR surgery with hamstring auto graft; (c) approximately four to 12 months post-surgery; (d) able to walk without pain or restriction, and (f) no other musculoskeletal conditions that could affect walking performance. Participants with swelling, pain or stiffness of the knee and body mass index exceeding $35 \mathrm{~kg} / \mathrm{m}^{2}$, and limb length discrepancy of more than two centimetres were excluded from the study. All participants were briefed about the study protocol, and gave written consent prior to measurements session. 


\subsection{Measurement Tool}

A three-dimensional (3D) motion analysis system (Motion Analysis Corporation, USA) which connected to six infrared motion-tracking cameras (Model Osprey) (Figure 1) was used to record the knee kinematics during gait. The cameras were positioned by using a floor reference grid to establish an identical recording distance from participant, which set at 120 $\mathrm{Hz}$ sampling rate and calibrated prior to measurement of each participant for every test session. The 3D motion analysis systems were synchronized with two force platforms (Model FP4060, Bertec Corporation, USA) (Figure 2) which is embedded into customized walking platform ( 6-meter) (Figure 3).

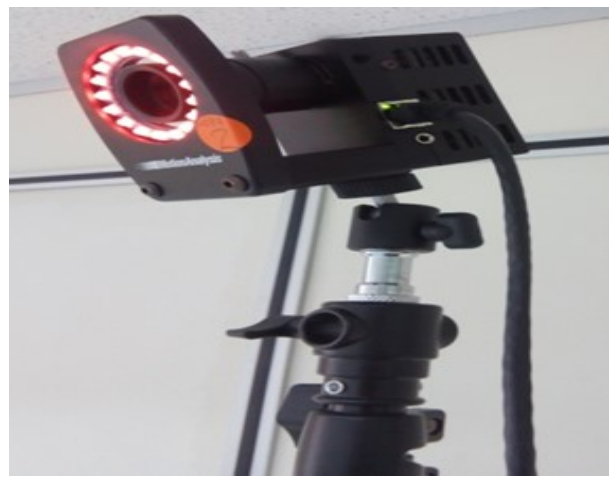

Fig.1. Osprey digital camera

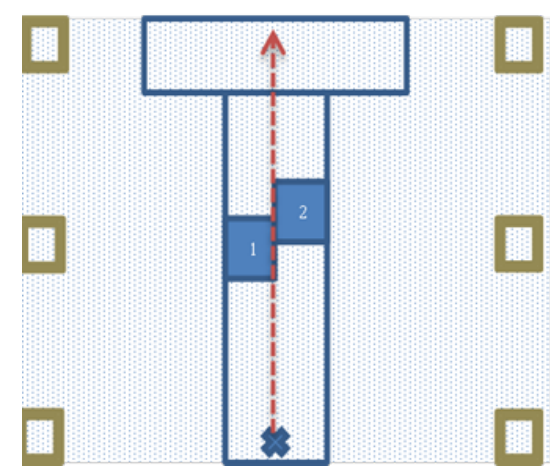

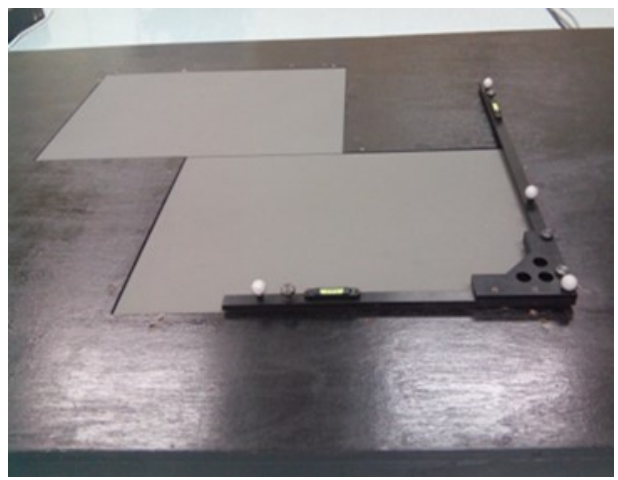

Fig.2. Embedded Force-plate FP4060-10

Fig.3. Laboratory set-up with customized walking platform. The red dashed line show a walking direction in gait analysis. The grey boxes represent camera position. The blue boxes represent force platform, and the $\mathrm{x}$ mark represent starting position (Please refer to online version for coloured lines). 
The force platform was set at $600 \mathrm{~Hz}$ sampling rate to record kinetic variables of ground reaction force during initial contact (IC) event of gait. The Global Coordinate System (GCS), which used for the measurement was based on the recommendation of International Society of Biomechanics (ISB) and force plate axes convention; $\mathrm{X}$ indicates medio/lateral direction, $\mathrm{Y}$ indicates anterior/posterior direction and $\mathrm{Z}$ axis represents upward/downward direction [16,17]. The right-handed inertial reference system were applied for both left and right body segments $[16]$.

\subsection{Measurement Procedure}

Prior to gait procedure and knee kinematic measurement, each participant was interviewed for demography details such as age, weight, and height and limb dominance. BMI was then calculated, followed by inspection of post-ACLR knee for presence of any swelling and pain. Limb length and quadriceps angles were assessed using conventional method to detect discrepancy between left and right side. Next, the inter-ASIS (anterior superior iliac spine) distance was measured to determine the centre of pelvic joint in 3D motion analysis system. Prior to use, the 3D motion analysis system and force platforms were calibrated for each individual. Participants were required to wear short tight and were barefooted throughout the gait assessment. First, thirty-two reflective markers using a modified Helen Hayes's markers set [18], were positioned over specified anatomical locations: i.e. trunk (left and right acromion

process, $7^{\text {th }}$ thoracic vertebra), pelvic (left and right ASIS, left and right PSIS, and sacrum), greater trochanter, upper and lower lateral aspect of thigh, medial and lateral femoral condyle, upper and lower lateral aspect of shank, lateral and medial malleolus at both legs, and left and right of foot (navicular head of $1^{\text {st }}$ metatarsal, head of $5^{\text {th }}$ metatarsal) for each participant (Figure 4).

A non-allergenic adhesive tape was used to secure the markers position over the identified bony landmarks. The thirty-two markers were used to capture the participant's static standing trial position and later designated as participant's neutral alignment. 


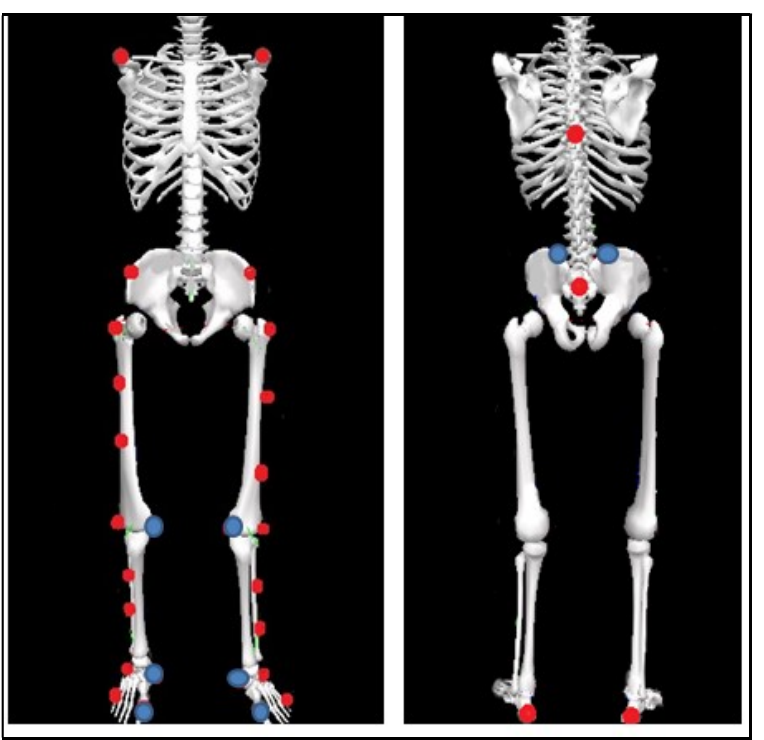

Fig.4(a)

Fig.4(b)
Fig.4. Skin markers location (a) in front, (b) back view. The blue markers were removed during gait analysis. (Please refer to online version for coloured picture).

Any recorded kinematic measures were referenced back to this position. In this static trial, each participant was instructed to stand on the force platform in their comfortable position with both feet at shoulder-width apart and without locking the knees. Then, following the static standing trial, eight anatomical markers were removed and the remaining 24 markers were used in the walking analysis [18]. Standardised protocol and verbal instruction was used through the measurement procedures; participants were instructed to walk bare-footed along the walking platform at a comfortable walking speed. Each patient was given at least two trials prior to the actual measurement to familiarize themselves with the walking procedure. At this stage, assessor marked the starting position of the gait on the walking platform, which was used to ensure that each foot make correct contact at specified area of force platform. A successful gait trial was confirmed when each foot make a correct contact with the force platform and positive feedback was given. To ensure normal walking pattern, participant were reminded not to look down and to walk at own walking pace throughout the sessions. Each participant was required to perform three successful gait trials. One-minute rest was given in between each trial. All participants were assessed twice with four hours interval between each test session. All measurements were conducted by one physiotherapist who was trained to use $3 \mathrm{D}$ motion analysis system. 


\subsection{Data Processing}

Motion capture data were collected using Cortex software (Model 1.5.3.1.1543, Motion Analysis Corporation, USA) and later exported to Visual 3D Profesional software (Version 5.0, C-Motion, USA) for further data reduction. The kinematic data were time-normalized to $100 \%$ of gait cycle. Raw data from Visual 3D software was later saved in text file and exported to Microsoft Excel 2010 (Microsoft Corporation, USA) for further analysis.

\subsection{Statistical Analysis}

All data was entered into statistical package for social sciences (SPSS) version 22 for analysis. The knee kinematic of interest was three-dimensional knee angles during the initial contact of gait. The mean and standard deviation of the angles were determined by calculating the average out of the three walking trials. Paired t-test was used to compare the obtained mean value between the first and the second measurement session for detection of systematic bias. The statistical significance was set at $\mathrm{p}$ value $<0.05$. The test-retest reliability level was determined by looking at the intraclass correlation coefficient (ICC); poor (ICC <0.4), moderate (ICC 0.4 to 0.8$)$ and excellent $(>0.8)[19,20]$.

\section{RESULTS}

Table 1 show characteristic of patient with ACLR knee. The mean (SD) age of the eight post-ACLR patients was 28 (4.0) years, with mean BMI of $26(4) \mathrm{kg} / \mathrm{m}^{2}$. Five out of the eight participants had ACLR surgery on the right knee. The measurement was conducted at an average of five months following ACLR surgery. The average walking speed of the patients in session one and two was $0.90 \mathrm{~m} / \mathrm{s}$ and $0.94 \mathrm{~m} / \mathrm{s}(\mathrm{ICC}=0.97)$, respectively.

Table 1. Characteristics of ACLR participants $(n=8)$

\begin{tabular}{cc}
\hline Characteristics & Mean (SD) \\
\hline Age (years) & $28.89(4.38)$ \\
Weight $(\mathrm{Kg})$ & $77.82(12.37)$ \\
Height $(\mathrm{m})$ & $1.70(0.04)$ \\
BMI $\left(\mathrm{kg} / \mathrm{m}^{2}\right)$ & $26.92(4.60)$ \\
\hline
\end{tabular}


Table 2 shows the calculated mean knee angles of the patients at each plane for the injured and non-injured knee in the first and second measurement sessions. The ICC values were excellent for the sagittal and transverse planes for the injured knee, 0.97(95\% CI: 0.84-0.99) and 0.96 (95\% CI: 0.83-0.99) respectively. Similar results were observed for the non-injured knee, with ICC above 0.90 (CI between 0.58 and 0.99). The test-retest reliability for knee angle measurements at frontal plane was found as moderate, with ICC $=0.44$ (CI -0.32 to 0.86 ). Paired t-test analysis found no significant difference in the mean value of knee angles at all the three planes of motion between the two measurement sessions (all $p>0.05$ ).

Table 2. Mean (SD) knee angles of the patients at each plane, $t$ (p) values, ICCs and $95 \%$ Confidence Interval for (a) injured knee and (b) non-injured knee

\begin{tabular}{lccccc}
\hline \multirow{2}{*}{ (a) Injured Knee } & $\begin{array}{c}\text { Session 1 } \\
\text { Mean(SD) }\end{array}$ & $\begin{array}{c}\text { Session 2 } \\
\text { Mean(SD) }\end{array}$ & t (p) Values & ICCs & 95\% CI \\
\hline Sagittal (x) & $-14.03(5.02)$ & $-13.42(4.9)$ & $-1.30(0.23)$ & 0.97 & $(0.840 .99)$ \\
Frontal (y) & $-3.06(6.50)$ & $-1.97(10.99)$ & $-0.32(0.75)$ & 0.44 & $(-0.32-0.86)$ \\
Transverse (z) & $1.72(20.03)$ & $-1.31(17.57)$ & $1.70(0.13)$ & 0.96 & $(0.83-0.99)$ \\
\hline \multirow{2}{*}{ (b) Non-injured Knee } & Session 1 & Session 2 & & & \\
& Mean(SD) & Mean(SD) & & & \\
\hline Sagittal (x) & $-6.25(11.83)$ & $-8.20(12.89)$ & $1.16(0.28)$ & 0.93 & $(0.68-0.99)$ \\
Frontal (y) & $1.82(5.76)$ & $-0.99(5.84)$ & $1.29(0.23)$ & 0.44 & $(-0.32-0.86)$ \\
Transverse (z) & $0.34(21.40)$ & $-0.99(29.38)$ & $0.32(0.75)$ & 0.90 & $(0.58-0.98)$ \\
\hline
\end{tabular}

\section{DISCUSSION}

In the current study, the test-retest reliability of knee angle measurement using 3D motion analysis system in multi-planes of motion during IC event of gait among post-ACLR male patients were assessed. The results indicate that the measurement technique has excellent test-retest reliability for both injured and non-injured knee at sagittal and transverse planes of motion, with ICC value for the sagittal plane higher than the value for the transverse plane which are consistent with results reported in other studies [10,12,21]. For example, Ferber and colleagues [21] who measured kinematics of the right knee during running in 20 recreational 
runners found higher reliability of measurements in the sagittal plane compared to frontal or transverse planes. The authors suggested markers reapplication, soft tissue artifact and individual physiologic variability as the contributing factors for the measurement variability. In another study, Labbe and colleagues [12] examined the effect of exoskeleton on knee kinematic measurement during walking among healthy individual. They also found that the test-retest reliability of knee angle measurement was higher for the sagittal plane than the other two planes. The current study findings support the findings of the two previous studies $[12,21]$ that indicate knee angle measurement using 3D motion analysis system is more reliable in the sagittal plane than other planes. In current study, the moderate test-retest reliability was found for the knee angle measurement at the frontal plane during initial contact of gait. This result is in agreement with the finding of previous work by Laroche and colleagues in the year 2011 [10]. They examined the knee angle of 23 patients with hip OA and reported lower reliability of knee frontal plane motion measurement during heel strike (ICC of OA and healthy sided, 0.69 and 0.79 , respectively) event after 30 recordings. The researchers stressed that the lower reliability value was due to the smaller available range of motion in the frontal of the knee compared to other planes [10].

In the literatures, several factors have been identified had threaten the accuracy of kinematics measurement using motion analysis system. Three main factors are marker placement, referenced static position, and physiological variability in each participant $[12,22,23]$. Ensuring accuracy of markers placement has been identified as a major challenge in movement analysis researchers [21,22]. To reduce the likelihood of placement errors, it is suggested that only trained persons should perform markers placement and conduct the measurement or test procedure [21]. Measurement by single assessor is recommended to reduce potential errors that could emerge due to differences in techniques between assessors [24]. Static positioning calibration for each participant is important to establish the participant's anatomical neutral joint alignment at which reference was made in measuring kinematics [22]. Increased between-test variability of frontal plane knee motion measurement could be due to lack of standardization in the calibration of static position calibration session. Knee lock position could be employed to provide maximal stability in upright position and reduce static position variability. However this is not a normal standing position, and does not 
represent participants' normal joint alignment, therefore is not a favourable strategy [25]. Another factor, which is physiologic variability in the participants, can be due to pathological condition or possible fatigue, which may occur during within day measurements and cause variation in gait pattern [26]. Some precaution can be taken to minimise fatigue such as providing a one-minute rest in between each trial and ensure measurement is repeated only after at least 4 hours [26].

The relatively small sample size in the study has limited the interpretation of the study findings. Hopkins [27] and Atkinson and colleagues [28] recommended that any reliability study should have a sample size of 40 or larger in order to obtain adequate precision. However, because this study used 3D motion analysis system in which the overall material cost is substantial, it is not possible to recruit up to 40 participants. The small number of study participants is consistent with other past studies of motion analysis in the literature [26,27]. Despite its limitation, the study findings shed some light on the approach to measure knee joint angles at multiple planes during gait. It is important for each study to investigate the measurement repeatability of adapted measurement protocol before it can be used in clinical or research activity. As proposed in a study by Kottner and colleagues, reliability studies act as quality assurance to provide sufficient information on the study methodology on specific population of interest [13]. Besides that, the current study also has a practical implication for therapist and researcher who involved in gait assessment. The practical implication of this finding is that by using a standardized measurement method, the ACLR rehabilitation team may obtain reliable multi-plane knee kinematic measurement, which further assists in accurate detection of knee changes in multiplanar movement following ACLR treatment session. The reliable assessment would enhance ACLR treatment plan for patient in acute and chronic stage of rehabilitation.

At present, the variability of multi-plane knee kinematic measured in different laboratories presented a mixed result. In particular, the information of variability of knee kinematic measurement in multi-planes is still lacking among ACLR male patient. Therefore, further studies focusing on reliability of multi-plane knee kinematic measurement for inter-trial, inter-session, and inter-laboratories are warranted to determine its potential use in clinical practice. It is well documented in the literature that measurement techniques to assess $3 \mathrm{D}$ joint 
rotation were potentially compromised by skin movement artifact [29] therefore any attempt to interpret the knee measurement data in frontal and transverse plane should be done cautiously. Finally, as there is no single statistical measurement is adequate to explain on reliability of measurements [13], future studies is recommended to add other measure of reliability such as standard error of measurement (SEM), Bland-Altman plot, and minimal detectable change (MDC) to strengthen the study findings.

\section{CONCLUSION}

The measurement of knee angle measurement in multi-planes of motion using 3D motion analysis system produced a moderate to excellent test-retest reliability among male patients following ACLR. The measurement protocol can be used in future studies or clinical setting using a similar population. In this study, participant recruitment was restricted to male patient only to negate the effect of gender factor. Further studies with larger participants are warranted.

\section{ACKNOWLEDGEMENTS}

This project received financial support from Ministry of Higher Education of Malaysia, via the Program Skim Latihan Akademik Bumiputera (SLAB). The authors wish to thank Dr. Leonard Joseph, Prof. Dr. Baharudin Hj. Omar and Dr. Johan Ahmad for their contribution in this study.

\section{REFERENCES}

[1] Janssen KW, Orchard JW, Driscoll TR, van Mechelen W. High incidence and costs for anterior cruciate ligament reconstructions performed in Australia from 2003-2004 to 2007-2008: time for an anterior cruciate ligament register by Scandinavian model? Scand J Med Sci Sports, 2012, 22(4), 495-50. doi:10.1111/j.1600-0838.2010.01253.x

[2] Flanigan DC, Everhart JS, Pedroza AD, Smith T, Kaeding CC. Fear of reinjury (kinesiophobia) and persistent knee symptoms are common factors for lack of return to sport after anterior cruciate ligament reconstruction. Arthroscopy, 2013, 29(8), 1322-1329. doi: 10.1016/j.arthro.2013.05.015

[3] Hosseini A, Li JS, Gill IV TJ, Li G. Meniscus injuries alter the kinematics of knees with anterior cruciate ligament deficiency. Orthop J Sport Med, 2014, 2(8), 2325967114547346. 
doi: $10.1177 / 2325967114547346$

[4] Leporace G, Metsavaht L, de Oliveira LP, Nadal J, Batista LA. Motor coordination during gait after anterior cruciate ligament injury: a systematic review of the literature. Rev Bras Ortop (English Edition), 2013, 48(4), 293-299. doi.org/10.1016/j.rboe.2012.07.008

[5] Webster KE, Feller JA. Alterations in joint kinematics during walking following hamstring and patellar tendon anterior cruciate ligament reconstruction surgery. Clin Biomech (Bristol, Avon), 2011, 26(2), 175-80. doi: 10.1016/j.clinbiomech.2010.09.011

[6] Gao B, Zheng NN. Alterations in three-dimensional joint kinematics of anterior cruciate ligament-deficient and -reconstructed knees during walking. Clin Biomech, 2010, 25(3), 222-229. doi: 10.1016/j.clinbiomech.2009.11.006

[7] Barenius B, Ponzer S, Shalabi A, Bujak R, Norlén L, Eriksson K. Increased risk of osteoarthritis after anterior cruciate ligament reconstruction: a 14-year follow-up study of a randomized controlled trial. Am J Sports Med, 2014, 42(5):1049-1057. doi: $10.1177 / 0363546514526139$

[8] Quatman CE, Quatman-Yates CC, Hewett TE. A "plane” explanation of anterior cruciate ligament injury mechanisms: a systematic review. Sport Med, 2010, 40(9):729-746. doi: $10.2165 / 11534950-000000000-00000$

[9] Kiapour AM, Quatman CE, Goel VK, Wordeman SC, Hewett TE, Demetropoulos CK.Timing sequence of multi-planar knee kinematics revealed by physiologic cadaveric simulation of landing: implications for ACL injury mechanism. Clin Biomech (Bristol, Avon), 29(1):75-82. doi: 10.1016/j.clinbiomech.2013.10.017

[10] Laroche D, Duval A, Morisset C, Beis JN, d'Athis P, Maillefert JF, et al.Test-retest reliability of 3D kinematic gait variables in hip osteoarthritis patients. Osteoarthr Cartil, 2011, 19(2):194-199. doi: 10.1016/j.joca.2010.10.024

[11] Fortin C, Nadeau S, Labelle H. Inter-trial and test-retest reliability of kinematic and kinetic gait parameters among subjects with adolescent idiopathic scoliosis. Eur Spine J, 2008, 17(2):204-216. doi: 10.1007/s00586-007-0469-9

[12] Labbe DR, Hagemeister N, Tremblay M, de Guise J.Reliability of a method for analyzing three-dimensional knee kinematics during gait. Gait Posture, 2008, 28(1):170-174. doi: 10.1016/j.gaitpost.2007.11.002 
[13] Kottner J, Audigé L, Brorson S, Donner A, Gajewski BJ, Hróbjartsson A, et al. Guidelines for Reporting Reliability and Agreement Studies (GRRAS) were proposed. Int J Nurs Stud, 2011, 48(6):661-671. doi: 10.1016/j.ijnurstu.2011.01.016

[14] Wünschel M, Wülker N, Müller O.Gender differences in tibio-femoral kinematics and quadriceps muscle force during weight-bearing knee flexion in vitro. Knee Surgery, Sport Traumatol Arthrosc, 2013, 21(11):2557-2563. doi: 10.1007/s00167-012-2082-7

[15] Nagano Y, Ida H, Akai M, Fukubayashi T. Gender differences in knee kinematics and muscle activity during single limb drop landing. Knee, 2007, 14(3):218-223. doi: $10.1016 / \mathrm{j}$. knee. 2006.11 .008

[16] Winiarski S, Rutkowska-Kucharska A. Estimated ground reaction force in normal and pathological gait. Acta Bioeng Biomech, 2009, 11(1):53-60.

[17] $\mathrm{Wu} \mathrm{G}$, Cavanagh PR. ISB recommendations in the reporting for standardization of kinematic data. J Biomech.1995, 28(10):1257-1261.

[18] Lyle MA, Valero-Cuevas FJ, Gregor RJ, Powers CM. Control of dynamic foot-ground interactions in male and female soccer athletes : females exhibit reduced dexterity and higher limb stiffness during landing. J Biomech, 2014, 47(2):512-517. doi: 10.1016/j.jbiomech.2013.10.038

[19] Kumar SN, Omar B, Htwe O, Joseph LH, Krishnan J, Esfehani AJ, et al. Reliability, agreement, and validity of digital weighing scale with MatScan in limb load measurement. J Rehabil Res Dev, 2014, 51(4):591-598. doi: 10.1682/JRRD.2013.07.0166

[20] Pollock C, Eng J, Garland S. Clinical measurement of walking balance in people post stroke: a systematic review. Clin Rehabil, 2011, 25(8):693-708. doi: $10.1177 / 0269215510397394$

[21] Ferber R, McClay Davis I, Williams DS, Laughton C. A comparison of within- and between-day reliability of discrete 3D lower extremity variables in runners. J Orthop Res., 2002, 20(6):1139-1145. doi: 10.1016/S0736-0266(02)00077-3

[22] Alenezi F, Herrington L, Jones P, Jones R.The reliability of biomechanical variables collected during single leg squat and landing tasks. J Electromyogr Kinesiol, 2014, 24(5):718-721. doi: 10.1016/j.jelekin.2014.07.007

[23] Akbarshahi M, Schache AG, Fernandez JW, Baker R, Banks S, Pandy MG. 
Non-invasive assessment of soft-tissue artifact and its effect on knee joint kinematics during functional activity. J Biomech, 2010, 43(7):1292-1301. doi: 10.1016/j.jbiomech.2010.01.002.

[24] Fairus FZ, Joseph LH, Omar B, Ahmad J, Sulaiman R. Intra-rater reliability and minimal detectable change of vertical ground reaction force measurement during gait and half-Squat tasks on healthy male adults. Malaysian J Med Sci, 2016, 23(2):21-27.

[25] Georgoulis AD, Ristanis S, Moraiti CO, Paschos N, Zampeli F, Xergia S, et al. ACL injury and reconstruction: clinical related in vivo biomechanics. Orthop Traumatol Surg Res, 2010, 96(8 Suppl):S119-28. doi: 10.1016/j.otsr.2010.09.004

[26] McGinley JL, Baker R, Wolfe R, Morris ME. The reliability of three-dimensional kinematic gait measurements: a systematic review. Gait Posture, 2009, 29(3):360-369. doi: 10.1016/j.gaitpost.2008.09.003

[27] Hopkins WG. 2000 Measures of reliability in sports medicine and science. Sport Med, 2000, 30(1):1-15

[28] Atkinson G, Nevill AM. Statistical methods for assssing measurement error (reliability) in variables relevant to sports medicine. Sport Med,1998, 26(4):217-238

[29] Nakagawa TH, Moriya ET, Maciel CD, Serrão FV. Test-retest reliability of three-dimensional kinematics using an electromagnetic tracking system during single-leg squat and stepping maneuver. Gait Posture, 2014, 39(1):141-6.doi: 10.1016/j.gaitpost.2013.06.011

\section{How to cite this article:}

Fairus F. Z., Nordin N. A. M. and Singh D. K. A. Test-retest reliability of knee kinematics measurement during gait with 3D motion analysis system. J. Fundam. Appl. Sci., 2017, 9(6S), 1236-1249. 